\title{
Self-organized criticality, long-time correlations, and the standard transport paradigm
}

\author{
John A. Krommes \\ Princeton University, P.O. Box 451, Princeton, New Jersey 08543-0451, USA
}

(November 12, 1999)

\begin{abstract}
Some aspects of low-frequency, long-wavelength fluctuations are considered. A stochastic model is used to show that power-law time correlations need not arise from self-organized criticality. A formula for the frequency spectrum of uncorrelated, overlapping avalanches is shown to be a special case of the spectral balance equation of renormalized statistical turbulence theory. It is argued that there need be no contradiction between the presence of long-time correlations and the existence of local transport coefficients.
\end{abstract}

PACS: 52.35.Ra

\section{INTRODUCTION}

Recently there have been serious attempts to introduce new physical and mathematical ingredients to the repertoire of tools available for calculating plasma transport. One research thread relates to the possible relevance of self-organized criticality ${ }^{1}$ (SOC), originally discussed in the context of plasma physics by Diamond and Hahm. ${ }^{2}$ Representative subsequent references include calculations on highly simplified SOC models by Newman and coworkers, ${ }^{3}$ reviews of the key ideas by Carreras et al. ${ }^{4}$ and Newman, ${ }^{5}$ simulations by Carreras et al. ${ }^{6}$ discussions of self-similarity properties of edge fluctuations by Carreras et al. ${ }^{7}$ experimental measurements of avalanche-like behavior by Politzer, ${ }^{8}$ and analysis of simulation data by Nevins. ${ }^{9}$

The theory of SOC is intended to introduce a new perspective to certain kinds of highly nonlinear phenomena. Various authors have found appealing the idea that in self-organized systems avalanches can be generated on all scales, leading to low-frequency, long-wavelength, selfsimilar correlations. This observation leads to a second thread relating to the possible significance of long-time correlations in certain experimentally observed time series, as reported for example by Carreras et al. ${ }^{10^{-13}}$ Carreras $^{11}$ suggested that "the measurements [on long-time tails] are consistent with the SOC paradigm of turbulent transport." (He cautioned "However, it does not prove that this model offers the only explanation... We are not aware of other dynamical mechanisms that may provide an alternative answer, but it may exist.") He also suggested ${ }^{12}$ that the existence of long-time tails may require that the "standard transport paradigm" (of local, diffusive transport) be abandoned. These suggestions motivated the present work.

The phrase "SOC paradigm" appears to have multiple connotations, with scenarios involving one or more of avalanches, power-law spectra, nearly marginal profiles with instability thresholds, strong intermittency, and nondiffusive motion. Confusion arises because none of those features are intrinsically linked to SOC, although any of them may have interesting consequences for transport and is worthy of study in its own right. A basic question is whether formalism and/or ideas specific to SOC need to be invoked in order to explain observations, or whether standard turbulence theory suffices.

Frequently SOC systems are said to exhibit self-tuning to a preferred nonequilibrium state with power-law (selfsimilar) spectra, thereby being distinguished from situations in equilibrium critical phenomena in which parameters must be carefully adjusted in order to achieve the critical state. However, it has been argued ${ }^{14}$ that true SOC behavior arises only in the limit that the forcing rate approaches 0 , that rate playing the role of a tuning parameter. The unusual limiting value of 0 forces one to worry about an interchange of limits. If a characteristic macroscopic time associated with avalanches is $\tau_{A}$ and the inverse of the forcing rate is $\tau_{f}$, then one has

$$
\tau_{f} \ll \tau_{A} \text { (conventional), } \quad \tau_{A} \ll \tau_{f} \text { (SOC). }
$$

Note that internal forcing due to microinstabilities probably obeys ordering (1a); that is, realistic systems are observed in the limit of highly overlapping "avalanches" (read "elementary excitations").

Another way of characterizing SOC regimes is with the concept of local rigidity, a measure of the importance of the threshold condition for linear instability, clearly discussed by Cafiero et al. ${ }^{15}$ SOC behavior with a power-law distribution of avalanches emerges in the limit of very large local rigidity. Further unifying remarks were made by Jensen. ${ }^{16}$

It would thus appear that pure SOC behavior lives in an asymptotic corner of parameter space. Realistically, one has neither infinitely slow forcing nor infinitely large local rigidity, so it is difficult to make a case for pure SOC. Therefore, attention has focused on qualitative features of some of the SOC models. For example, it has been suggested that submarginal (linearly stable) mean profiles are important, in that fluctuations that transiently induce local instability might lead to intermittent states with avalanches, long-time correlations, and nonlocal transport.

A general goal is to understand the connections between the physical and mathematical descriptions of SOC and the better-understood intuition and formalism of renormalized statistical turbulence theory. That is by no means fully accomplished in the present paper; however, some introductory steps are taken, mostly focused on the interpretation 
of the so-called spectral balance equation employed ubiquitously in statistical turbulence theory. That general equation applies to all regions of parameter space; for example, it embraces both of the ordering regimes (1a) and (1b) and thus copes with the likelihood that the observed turbulent states lie in the regime of strongly overlapping avalanches. I consider the possibility that long-time correlations of the kind observed experimentally can have relatively prosaic origins unrelated to esoteric (e.g., submarginal or rigidthreshold) SOC scenarios, and I explain why long-time correlations do not inevitably lead to the breakdown of conventional transport theory. Specifically, in Sec. II I describe a stochastic model that possesses long-time correlations yet lacks significant features of some SOC scenarios discussed in the plasma-physics literature. In Sec. III I show that a well-known SOC formula for the spectral density of overlapping avalanches is a special case of the spectral balance equation. In Sec. IV I argue that there need be no contradiction between the presence of long-time correlations and the existence of local transport coefficients. To do so, I emphasize the distinction between Eulerian correlation functions (easily measured) and Lagrangian correlation fucntions (relevant to transport), illustrating with some analysis based on Hasegawa-Mima and guiding-center dynamics. Sec. V contains a brief discussion.

\section{A STOCHASTIC MODEL WITH LONG-TIME CORRELATIONS}

To a person without prior theoretical or experimental knowledge of the internal workings of a turbulent confinement device, the machine can profitably be viewed as a black box that emits certain diagnostic signals that can be studied. A sufficiently complete set of signals ultimately enables one to reconstruct all internal details. However, an incomplete set of signals leads to ambiguity of interpretation. It can be argued that in the context of long-time correlations the possibility of such ambiguity has not been adequately emphasized in the literature. Two questions are: (1) Are long-time tails on correlation functions invariably associated with SOC? (2) Do such tails inevitably imply nonlocal or nondiffusive dynamics? The answers affect the choice of ingredients for a theoretical description.

In order to shed light on such questions, one can construct a mathematical "black box" (in the form of a stochastic model) that emits signals similar, in some highly simplified sense, to those seen experimentally. The idea, illustrated schematically in Fig. 1, is to replace the highly complicated and self-consistent plasma dynamics with a much simpler stochastic differential equation to which certain statistical properties are assigned. Boundary conditions are imposed such that a turbulent flux $\Gamma$ (say of a scalar field called "temperature" $T$ ) with nonzero mean flows through the system. If it is simple enough, the model can be solved numerically (thereby avoiding possible controversies about analytical approximations), and various properties such as the two-time (Eulerian) correlation function $C_{E}(\tau)$ of the flucuations $\delta T$ of $T$ at a fixed position can be subsequently measured. The goal is to show that $C_{E}(\tau)$ can possess long-time tails even when the underlying dynamics of the stochastic model look nothing like the discrete sandpile dynamics frequently used to illustrate SOC phenomena and do not obey the ordering (1b). Of course, this proves nothing about the actual experiments; it merely emphasizes the substantial ambiguity that exists with a restricted set of measurements. However, it is not implausible that the real plasma has features in common with an appropriately constructed stochastic model.

Although models of any dimensionality can be constructed in principle, in practice it is highly convenient to work in one dimension (1D) in order that direct numerical simulations can be done and adequate statistics collected. (1D stochastic models with multiplicative statistics effectively emulate higher-dimensional systems and are more realistic than the additively forced 1D Burgers equation that is often discussed. ${ }^{2}$ ) A particular 1D model of turbulent thermal transport was recently studied by Krommes and Ottaviani. ${ }^{17}$ They considered a passive advection model for a scalar field $T(x, t)$ in which the advecting velocity $\widetilde{V}(x, t)$ was a Gaussian variable with unit strength (and no threshold) and specified autocorrelation time $\tau_{\mathrm{ac}}$ and length $L_{\mathrm{ac}}$. Those parameters could be taken to be small, in which case local transport (with supermarginal profiles ${ }^{18}$ ) was recovered; nevertheless, Eulerian time correlations of $\delta T$ (measured, say, at the center of the box) decayed algebraically, $C_{E}(\tau) \sim \tau^{-1 / 2}$. A simple analytical argument that predicted the exponent of $-1 / 2$ was also given. The absence of a threshold means that conventional avalanches do not occur, as can be verified by measuring a space-time event distribution following a procedure similar to that of Nevins. ${ }^{9}$

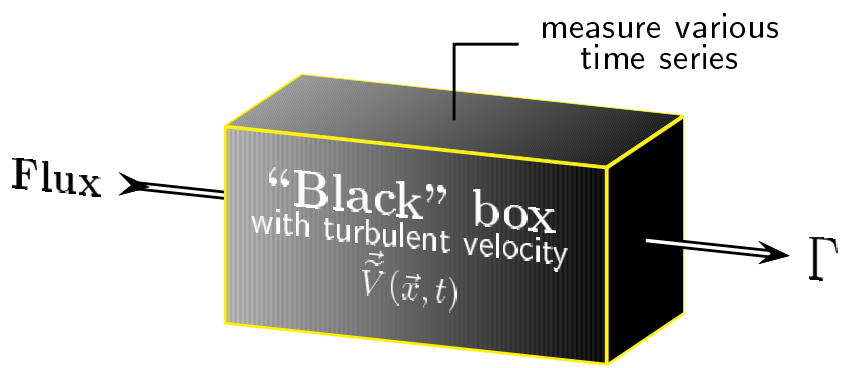

FIG. 1. A "black box," in the form of a stochastic model that introduces a statistically specified advection velocity $\widetilde{\boldsymbol{V}}(\boldsymbol{x}, t)$, can be used to illustrate that long-time correlations need not arise from SOC and need not be associated with nonlocal transport.

This behavior-local, supermarginal, non-avalanching transport but long-time tails - emphasizes the difficulty of identifying the details of underlying dynamics from a restricted set of macroscopic measurements. A proper formalism should cope with all of these facets, yet should not eliminate the possibility of avalanches at the outset. One is therefore motivated to consider the relationship of formulas derived in specific SOC contexts to more general ones. 


\section{THE WEIGHTED LIFETIME DISTRIBUTION AND THE SPECTRAL BALANCE EQUATION}

In SOC theory, one of the central results for the frequency spectra of avalanches is a formula by Jensen ${ }^{19}$ that relates the power spectrum of the total dissipation rate to a particular weighted distribution $\Lambda$ of avalanche lifetimes. In general turbulence theory, the most important relationship governing frequency spectra is the so-called spectral balance equation. I show in the present section how these formalisms are related, thus establishing a general link between the superficially distinct lines of research and emphasizing the general validity of spectral balance.

Consider a generic (mathematical) sandpile in which an avalanche is excited by dropping one extra grain of sand on a random lattice site at some integer time $t_{i}$. For that primitive avalanche, the dissipation rate for any subsequent time $t_{j}$ is defined as the total number of sites that topple during the $j$ th time interval. If grains are dropped on random sites at each integer time, a signal $J(t)$ can be defined as the total dissipation rate summed over all avalanches. Then Jensen's formula for the spectral power density $C_{J}(\omega)=\int_{-\infty}^{\infty} d \tau e^{i \omega \tau} C_{J}(\tau)$ of the autocorrelation function $C_{J}(\tau)$ of $J$ is

$$
C_{J}(\omega)=2 \nu \int_{0}^{\infty} d T \frac{[1-\cos (\omega T)]}{\omega^{2}} \Lambda(T),
$$

where $\Lambda(T) \doteq T^{-2} \int_{0}^{\infty} d S P(S, T) S^{2}, P(S, T)$ being the joint probability density function (PDF) for total signal $S$ and lifetime $T$ in an avalanche.

I recall the derivation of this formula, closely following Appendix D of Ref. 19. Let the time signal of an individual avalanche $A$ be $\mathcal{J}_{A}(t)$. The total measured signal $J(t)$ is assumed to be the sum of individual signals started at random times: $J(\tau)=\sum_{t_{i}<t} \mathcal{J}_{A_{i}}\left(t-t_{i}\right)$. The individual avalanches are assumed to be uncorrelated for $i \neq j$ and to have zero mean. Then with $\tau \doteq t-t^{\prime}$ being the usual time difference in a two-point measurement, one has for $\tau>0 C_{J}(\tau)=\left\langle\sum_{t_{j}<t-\tau} \mathcal{J}_{A_{j}}\left(t-t_{j}\right) \mathcal{J}_{A_{j}}\left(t-\tau-t_{j}\right)\right\rangle$. On the average, avalanches are assumed to occur with rate $\nu$. Upon introducing the mean time step $\Delta t=\nu^{-1}$ between avalanches, one can view the sum over $j$ as a Riemann approximation to a time integral; thus $C_{J}(\tau)=$ $\nu \int_{0}^{\infty} d t\left\langle\mathcal{J}_{A}(t+|\tau|) \mathcal{J}_{A}(t)\right\rangle$, where the remaining average is over the characteristics (size, lifetime, etc.) of the random avalanches. For a crude but apparently adequate description, Jensen characterizes each signal $\mathcal{J}$ by its lifetime $T$ and integrated signal (area under the curve) $S$ and adopts the tophat approximation

$$
\mathcal{J}_{A}(t)=\mathcal{J}_{S, T}(t)= \begin{cases}S / T & \text { for } t \in(0, T) \\ 0 & \text { otherwise }\end{cases}
$$

Then, with $H(t)$ being the unit step function,

$$
C_{J}(\tau)=\nu \int_{0}^{\infty} d t \int_{0}^{\infty} d T \int_{0}^{\infty} d S P(S, T)(S / T)^{2}
$$

$$
\begin{aligned}
\times[H(t & +|\tau|+T)-H(t+|\tau|)][H(t+T)-H(t)] \\
& =\nu \int_{|\tau|}^{\infty} d T \int_{0}^{\infty} d S P(S, T)(S / T)^{2}(T-|\tau|) .
\end{aligned}
$$

It is now straightforward to Fourier transform Eq. (4b) and recover Eq. (2) after two integrations by parts.

Now let us consider the relation of Eq. (2) to the spectral balance equation of statistical turbulence theory, which describes the balance between forcing and dissipation. For stationary fluctuations, one form of that equation is ${ }^{20,21}$

$$
C(\omega)=|R(\omega)|^{2} F(\omega),
$$

where $R$ is the mean infinitesimal response function and $F$ is the covariance of the effective incoherent noise driving the fluctuations. In general, $F$ consists of both internal and external parts: $F=F^{(\mathrm{int})}+F^{(\mathrm{ext})}$. Further discussion and examples of the use of Eq. (5) can be found in Refs. 22 and 23. Perhaps the easiest way to appreciate it is to note that it is compatible with the formal solution of the Langevin representation

$$
R^{-1} \psi=\widetilde{f}=\widetilde{f}^{(\mathrm{int})}+\widetilde{f}^{(\mathrm{ext})}, \quad R^{-1} \doteq \partial_{t}+\Sigma \star,
$$

where $\star$ denotes convolution $\left[\right.$ so $\left.R^{-1}(\omega)=-i\left(\omega+i \Sigma_{\omega}\right)\right]$, $\Sigma$ includes both linear physics and the (statistically sharp) turbulent damping, and $\widetilde{f}$ is the total random forcing. The statistics of $\widetilde{f}^{\text {(int) }}$ must be intimately related to $\Sigma$ in such a way that energy is conserved by the nonlinear interactions. A Langevin representation of the direct-interaction approximation (DIA) was given in Refs. 24 and 25; for related discussion, see Refs. 26 and 22 .

A special case of Eq. (5) can be found in the classical Langevin equation ${ }^{27} \dot{v}+\nu v=a(t)$, where

$$
F(\tau) \doteq\langle a(t+\tau) a(t)\rangle=2 D_{v} \delta(\tau), \quad F(\omega)=2 D_{v}
$$

Here the velocity-space diffusion coefficient $D_{v}$ is related to $\nu$ and the steady-state velocity fluctuation level $\mathcal{I}=v_{\mathrm{t}}^{2}$ via Einstein's relation $D_{v}=v_{\mathrm{t}}^{2} \nu$. The steady-state solution for the velocity covariance is

$$
C(\tau)=v_{\mathrm{t}}^{2} e^{-\nu|\tau|}, \quad C(\omega)=\left(\omega^{2}+\nu^{2}\right)^{-1} 2 \nu v_{\mathrm{t}}^{2},
$$

which can be written in the form (5) by identifying $\nu$ with $\Sigma_{\omega}$ and recalling Eq. (7b). Note that the damping rate $\nu$ can also be interpreted as the steady-state forcing rate $\nu=F(\omega) / \mathcal{I}$.

In the classical Langevin example, the parameters $\nu$ and $\mathcal{I}$ (or $\nu$ and $F$ ) are given parameters; although they ultimately depend on microscopic noise, the details of that noise are not "opened up" by the coarse-grained Langevin description. More generally, however, Eq. (5) is a nontrivial self-consistent integral equation (both $\Sigma$ and $F^{\text {(int) }}$ depend on $C$ ) that determines the two-point statistics of the turbulent noise. Imagine exciting a response in a nonlinear system (such as a sandpile) by external forcing $\delta \widetilde{f}^{\text {(ext) }}$. If $\delta \widetilde{f}^{(\mathrm{ext})}$ were infinitesimal, Green's solution would be 


$$
\delta \psi(t)=\int_{-\infty}^{t} d \bar{t} \widetilde{R}(t ; \bar{t}) \delta \widetilde{f}^{(\mathrm{ext})},
$$

where $\widetilde{R}$ is random to the extent that the undisturbed system is stochastic. Although the properties of the undisturbed system can be assumed to be independent of the external forcing, so for first-order response the covariance of $\delta \widetilde{f}^{\text {(ext) }}$ can be factored from the covariance of Eq. (9), one is not led to Eq. (5) with $F=F^{\text {(ext) }}$ because $\langle\widetilde{R} \widetilde{R}\rangle \neq R R$. Moreover, for finite, possibly large forcing the causal response $\delta \psi\left[\delta \widetilde{f}^{(\mathrm{ext})}\right]$ is a functional (indicated by the bracket notation) containing terms of all orders in $\delta \widetilde{f}^{(\mathrm{ext})}$, so the statistical average $\left\langle\delta \psi\left[\delta \widetilde{f}^{(\text {ext })}\right] \delta \psi\left[\delta \widetilde{f}^{(\text {ext })}\right]\right\rangle$ is extremely difficult to perform. The beauty of Eq. (5) is that it presents that average in terms of factored infinitesimal response functions (even though $\delta \widetilde{f}^{\text {(ext) }}$ need not be infinitesimal), making up the difference with the covariance $F^{(\mathrm{int})}$ of the internally produced noise. Of course, it may be very difficult to determine $F^{\text {(int) }}$; however, the DIA and related Markovian approximations ${ }^{28}$ lead to workable formulas.

Equation (5) permits arbitrary correlations between the fluctuations at different times. It must therefore be a generalization of Jensen's result (2). Indeed, one can process Eq. (5) to obtain a formula for a generalized weighted lifetime distribution. Consider first the interpretation of the integral $\mathcal{I} \doteq \int_{0}^{\infty} d T \Lambda(T)=\Lambda(\omega=0)$ that appears in Eq. (2). One has $\mathcal{I}=\int_{0}^{\infty} d T \int_{0}^{\infty} d S P(S, T)(S / T)^{2}$. Note that $S / T=A$, where $A$ is the amplitude of a primitive avalanche in the tophat approximation. Upon using $\{A, T\}$ instead of $\{S, T\}$ as variables and employing the normalization properties of a joint $\mathrm{PDF}$, one has $\mathcal{I}=\int_{0}^{\infty} d T \int_{-\infty}^{\infty} d A \widehat{P}(A, T) A^{2}=\int_{-\infty}^{\infty} d A \widehat{P}(A) A^{2}=\left\langle A^{2}\right\rangle$. Thus $\mathcal{I}$ is just the mean-square level of the fluctuations. It is then natural to consider a lifetime distribution $\widehat{\Lambda}$ normalized to that level. Since Jensen assumed uncorrelated avalanches, the $\nu$ in formula (2) is completely analogous to the $\nu$ in the classical Langevin example. If one identifies $\nu \rightarrow F / \mathcal{I}$, then one finds $[1-\widehat{\Lambda}(\omega)] / \omega^{2} \rightarrow|R(\omega)|^{2}$. Thus

$$
\widehat{\Lambda}(\omega)=1-\omega^{2}|R(\omega)|^{2} .
$$

If one assumes that $\Sigma$ is nonzero as $\omega \rightarrow 0$ (there is at least classical dissipation), then as a consistency check one can readily verify that $\lim _{\omega \rightarrow 0} \widehat{\Lambda}(\omega)=1, \lim _{\omega \rightarrow \infty} \widehat{\Lambda}(\omega)=0$.

It is useful to check that formula (10) makes physical sense. For purposes of illustration, I shall temporarily assume that $\Sigma_{\omega}$ is real. (Usually the causal function $\Sigma_{\omega}$ contains an imaginary part; the Kramers-Kronig relations must be respected.) At linear order, that means that waves are assumed to be absent. One then finds the esthetically pleasing result $\widehat{\Lambda}(\omega)=\Sigma_{\omega}^{2} /\left(\omega^{2}+\Sigma_{\omega}^{2}\right)$. This formula is a generalization of the characteristic function (Fourier transform) of the PDF for an event in a Poisson process. Thus, if one lets $\Sigma_{\omega} \rightarrow \nu$ (a constant), then $\widehat{\Lambda}(\tau)=\frac{1}{2} \nu e^{-\nu|\tau|}$, which is just $\frac{1}{2} d P(\tau) / d \tau$, where $P(\tau)=1-e^{-\nu \tau}$ is the probability of having at least one event (occurring with mean frequency $\nu)$ in the time interval $(0, \tau)$. The factor of $1 / 2$ accounts for the fact that $\tau$ may be negative.

Jensen showed that if $\widehat{\Lambda}(T) \sim T^{a} e^{-\nu T}$, then $C_{J}(\omega) \sim$ $\omega^{-(3+a)}(a \leq-1)$ or $\omega^{-2}(a \geq-1)$; he noted that for the cases studied one invariably has $a>-1$, leading to an $\omega^{-2}$ spectrum of the dissipation rate. (It is a misconception that all SOC-related signals have a spectrum close to $\omega^{-1}$.) Provided that the relevant Fourier transforms exist, Eq. (10) shows that $R(\omega)$ and $\widehat{\Lambda}(\omega)$ [or $\Sigma_{\omega}$ and $\widehat{\Lambda}(\omega)$ ] carry the same information. Given a $\widehat{\Lambda}$, the determination of $\Sigma_{\omega}$ is not immediate because of the Kramers-Kronig relations, but that is never necessary in practice, as one need never introduce the weighted lifetime distribution. In fact, Eq. (5) provides a more direct route to the frequency dependence of $C(\omega)$ because $\Sigma_{\omega}$ is a natural object in statistical theory. Nonanalytic frequency dependence of $\Sigma_{\omega}$, which can arise from certain kinds of nonexponential time decay of $\Sigma(\tau)$, can in principle give rise to frequency spectra different from $\omega^{-2}$. The balance equation also shows that nontrivial $\omega$ dependence of $F(\omega)$ can influence the result. A difficulty is that sufficiently unusual (e.g., fractal or intermittent) nonlinear behavior may not be properly captured by low-order statistical closures. But the balance equation itself holds even in the presence of strong intermittency.

So far the discussion has focused on purely temporal signals and the associated frequency spectra. But the spectral balance representation also permits a ready generalization to include spatial fluctuations and wave-number spectra. In the next section I shall show how particular superpositions of wave-number amplitudes can lead to decidedly nontrivial time dependence even when the behavior of an individual Fourier component is benign. Such possibilities further complicate the interpretation of long-time tails from black-box measurements of the type discussed in Sec. II.

\section{CORRELATIONS AND THE STANDARD TRANSPORT PARADIGM}

With a general theory of spectral balance in hand, I now turn to a discussion of some issues related to the calculation of turbulent transport coefficients. I recall Carreras' suggestion ${ }^{12}$ that in the face of long-time tails the "standard transport paradigm" might have to be abandoned. Although this remains a possibility, I shall argue that the conclusion is not inevitable.

It is difficult to find a comprehensive discussion of transport paradigms in the literature. The cleanest scenario is to envision turbulent noise with short wavelengths and correlation times, and to inquire about transport at long wavelengths and times. Then transport coefficients are well defined and can be estimated by simple random-walk considerations. This familiar situation can be illustrated by the stochastic passive advection equation $\partial_{t} \psi(\boldsymbol{x}, t)+\tilde{\boldsymbol{V}}(t)$. $\boldsymbol{\nabla} \psi=0$, where $\tilde{\boldsymbol{V}}$ is a centered Gaussian time series. One can make analytical progress by assuming that $\widetilde{\boldsymbol{V}}$ is independent of $\boldsymbol{x}$; then one can Fourier transform to obtain 


$$
\partial_{t} \psi(t)+i \widetilde{\omega}(t) \psi=0
$$

where $\psi(t) \equiv \psi_{\boldsymbol{k}}(t)$ and $\widetilde{\omega}(t) \doteq \boldsymbol{k} \cdot \widetilde{\boldsymbol{V}}(t)$. Since $\widetilde{\omega}$ is a centered Gaussian, it is uniquely specified by its covariance $S\left(t, t^{\prime}\right) \doteq\left\langle\delta \widetilde{\omega}(t) \delta \widetilde{\omega}\left(t^{\prime}\right)\right\rangle$, which is commonly taken to be $S(\tau)=\beta^{2} e^{-|\tau| / \tau_{\text {ac }}}$. The unique dimensionless parameter for this problem is then the Kubo number ${ }^{29} \mathcal{K} \doteq \beta \tau_{\text {ac }}$, which is a measure of the slowness of the velocity fluctuations. In terms of $\bar{\tau} \doteq \beta \tau, S(\bar{\tau})=e^{-|\bar{\tau}| / \mathcal{K}}$.

Equation (11) is exactly solvable ${ }^{30-32,21}$ and is also amenable to various statistical closures. For $\mathcal{K} \ll 1$, quasilinear theory is an adequate description for coarse-grained times long compared to $\tau_{\mathrm{ac}}$. The quasilinear approximation to Eq. (11) is $\partial_{t}\langle\psi\rangle+\eta\langle\psi\rangle=0$, where $\eta=\beta^{2} \tau_{\mathrm{ac}}=k^{2} D_{\mathrm{QL}}$ and $D_{\mathrm{QL}} \doteq \bar{V}^{2} \tau_{\mathrm{ac}}$, a conventional random-walk formula. For small $k$, one sees that the consequence of rapid velocity fluctuations is to set up a slow (hydrodynamic) diffusion mode with decay time $\left(k^{2} D_{\mathrm{QL}}\right)^{-1}$. This result is very well known. However, it is useful to consider this behavior from the point of view of the exact correlation function of $\psi$, which is $C(\tau)=\exp \left(-\mathcal{K}^{2}\left(|\bar{\tau}| / \mathcal{K}-1+e^{-|\bar{\tau}| / \mathcal{K}}\right)\right)$. For $\bar{\tau} \gg 1\left(\tau \gg \tau_{\mathrm{ac}}\right)$, one has $C(\tau) \rightarrow e^{-\mathcal{K}|\bar{\tau}|}=e^{-\beta^{2} \tau_{\mathrm{ac}}|\tau|}$, in agreement with the solution of the quasilinear approximation. Compare the $\mathcal{K}$ dependence of $C$ (an exponent of $-\mathcal{K} \bar{\tau}$; slow decay for $\mathcal{K} \ll 1$ ) with that of the underlying $S$ (an exponent of $-\bar{\tau} / \mathcal{K}$; rapid decay). Now suppose that Eq. (11) describes an actual physical system and that one makes direct diagnostic measurements of the time correlations of $\psi$, say by inserting a probe. Even if they decay slowly $(\mathcal{K} \ll 1)$, clearly one is not justified in inferring that a local description of transport does not exist; indeed, the opposite is true for this model. This point is trivial, but it emphasizes that the nature of the variable whose time correlations are being analyzed matters.

The above model fails to capture the essence of real turbulent systems in one important respect: in general, the turbulent velocity depends importantly on $\boldsymbol{x}$. With $\widetilde{\boldsymbol{V}}=\tilde{\boldsymbol{V}}(\boldsymbol{x}, t)$, useful exact solutions for the $\psi$ statistics are not forthcoming and one must resort to numerical simulations, statistical closure approximations, or general considerations based on spectral balance (see below). In so doing, it is important to keep in mind the distinction between Eulerian and Lagrangian correlation functions. In particular, whereas it is typically Eulerian correlation functions that are readily measured experimentally, it is Lagrangian correlation functions that are relevant to the calculation of transport coefficients. The behavior of those two classes of functions can be very different, as I will discuss.

In early seminal work, G. I. Taylor proved ${ }^{33}$ that a turbulent velocity field $\boldsymbol{u}(\boldsymbol{x}, t)$ gives rise to a particle diffusion coefficient $D$ whose value is $D=\int_{0}^{\infty} d \tau C_{L}(\tau)$, where $C_{L}$ is the Lagrangian correlation function. With $V$ being any Cartesian component of $\boldsymbol{u}$, one has (assuming spatially homogeneous and temporally stationary statistics)

$$
C_{L}(\tau) \doteq\langle\delta V(\boldsymbol{x}(t+\tau), t+\tau) \delta V(\boldsymbol{x}(t), t)\rangle .
$$

The well-known difficulty ${ }^{34}$ of evaluating formula (12), hence $D$, is that the random trajectory $\boldsymbol{x}(t)$ is unknown.

The most general Eulerian correlation function

$$
C_{E}\left(\boldsymbol{x}, t, \boldsymbol{x}^{\prime}, t^{\prime}\right) \doteq\left\langle\delta V(\boldsymbol{x}, t) \delta V\left(\boldsymbol{x}^{\prime}, t^{\prime}\right)\right\rangle
$$

depends on two space and two time points, specified independently. This function is relatively easy to measure experimentally, e.g. by inserting probes (at least in principle). A reduced function depending only on time difference can be defined by specializing to equal space points: $C_{E}(\tau) \doteq C_{E}\left(\boldsymbol{x}, t, \boldsymbol{x}, t^{\prime}\right)$. In general, $C_{E}(\tau)$ behaves radically differently from $C_{L}(\tau)$. To illustrate this, consider $1 \mathrm{D}$ for simplicity and suppose that $\psi(x, t)=X(x) T(t)$, where the random functions $X$ and $T$ are statistically independent and the two-time correlation function $\mathcal{T}$ of $T$ is arbitrary. For spatially homogeneous statistics, one has

$$
C_{E}(\tau)=\left\langle X^{2}\right\rangle\langle T(t+\tau) T(t)\rangle=\text { const. } \times \mathcal{T}(\tau),
$$

so $C_{E}$ decays slowly if $\mathcal{T}$ does and can easily be nonintegrable. However, that need not be the case for the Lagrangian function because $x(t)$ evolves randomly. As a primitive illustration, suppose that $x(t)=x+v t$, where $v$ has a Gaussian distribution of width $\bar{v}$. Consider for definiteness a single Fourier mode with random phase $\theta$ uniformly distributed on $[0,2 \pi)$, namely $X(x)=\cos (k x+\theta)$. One readily calculates that

$$
C_{L}(\tau)=\frac{1}{2}\langle\cos (k v \tau)\rangle \mathcal{T}(\tau)=\frac{1}{2} e^{-k^{2} \bar{v}^{2} \tau^{2} / 2} \mathcal{T}(\tau) .
$$

For algebraic or even pure exponential decay of $\mathcal{T}(\tau)$, the decay of $C_{L}$ is dominated at long times by the streaming contribution. More realistic evolution laws for $x(t)$ need not change this conclusion if the motion is random. Additional decay emerges when a spectrum of modes is considered.

Admittedly, the factored form assumed above likely does not hold in practice. Determining the precise spectral representation of a self-consistent velocity field is very difficult and requires numerical simulations or detailed statistical closure calculations. However, some general remarks about Eulerian and Lagrangian correlations can be made by considering some robust aspects of renormalized turbulence theory. An exact formula for $C_{E}(\tau)$ is [cf. Eq. (5)]

$$
C_{E}(\tau)=\int \frac{d \boldsymbol{k}}{(2 \pi)^{d}} \int_{-\infty}^{\infty} \frac{d \omega}{2 \pi} e^{-i \omega \tau}|R(\boldsymbol{k}, \omega)|^{2} F(\boldsymbol{k}, \omega),
$$

where $d$ is the dimension of space. Some attempts to analyze this formula were made in Ref. 17. It was concluded that algebraic tails for $C_{E}(\tau)$ followed with only mild assumptions; the key was to argue that the integral was dominated by the small $k$ 's and $\omega$ 's. Here I shall illustrate such behavior by employing, instead of the proper solution of the balance equation (say in the DIA), the so-called selfconsistent field approximation ${ }^{35,36}$

$$
C_{\boldsymbol{k}}(\tau)=R_{\boldsymbol{k}}(\tau) C_{\boldsymbol{k}}(\tau=0) \quad(\tau>0) .
$$

In thermal equilibrium Eq. (17) is an exact statement of the fluctuation-dissipation theorem, ${ }^{37,38}$ but it has qualitative 
validity in general and is also closely related to Langevin representations of successful Markovian closures. ${ }^{28}$ Then

$$
C_{E}(\tau)=\frac{\Omega_{d}}{(2 \pi)^{d}} \int_{0}^{\infty} d k k^{d-1} R_{k}(\tau) C_{k} \quad(\tau>0),
$$

where $\Omega$ is the solid angle and spatial homogeneity was used. If one reasonably assumes that $C_{k}$ is well behaved as $k \rightarrow 0$ and if one also assumes that $R_{k}(\tau) \sim e^{-k^{2} D \tau}$ in that same limit, then a stationary-phase evaluation of Eq. (18) for large $\tau$ leads to the asymptotic scaling

$$
C_{E}(\tau) \sim \tau^{-d / 2} .
$$

(For $d=1$, this agrees with the $\tau^{-1 / 2}$ behavior found for the stochastic model discussed in Sec. II.) Different assumptions about $R_{k}(\tau)$ lead $^{17}$ to different decay exponents, although the power-law behavior is preserved.

The formula (19) for the Eulerian function is reminiscent of the famous result of Alder and Wainwright ${ }^{39}$ for the decay of the Lagrangian velocity autocorrelation function in equilibrium many-body theory. As applied to $C_{L}$, it was rediscovered in plasma physics by Krommes and Oberman ${ }^{40}$ in the context of convective cells in 2D, strongly magnetized plasmas. The significance of a $\tau^{-d / 2}$ decay of $C_{L}$ is that it is nonintegrable for $d \leq 2$, in which case local transport coefficients do not exist.

Although in the examples cited in the previous paragraph the Eulerian and Lagrangian functions decay asymptotically at the same rate, that is not the case in general. A Lagrangian function can be integrable (and a local transport coefficient well defined) even when the associated Eulerian function is nonintegrable. To see that, one could analyze the time dependence of the mass operator. If the dependent variable is expected to diffuse at long wavelengths (as would be the case for a passively advected scalar, for example), then to within a factor of $k^{2} \Sigma_{\boldsymbol{k}}(\tau)$ is the relevant integrand whose time integral determines the turbulent transport coefficient $\mu$ in the hydrodynamic limit, namely ${ }^{41}$

$$
\mu=\int_{0}^{\infty} d \tau C_{L}(\tau), \quad C_{L}(\tau)=\lim _{k \rightarrow 0} k^{-2} \Sigma_{\boldsymbol{k}}(\tau) .
$$

For example, in the DIA

$$
\Sigma_{\boldsymbol{k}}(\tau)=-\sum_{\Delta} M_{\boldsymbol{k} \boldsymbol{p} \boldsymbol{q}} M_{\boldsymbol{p} \boldsymbol{k} \boldsymbol{k}}^{*} R_{\boldsymbol{p}}(\tau) C_{\boldsymbol{q}}(\tau) .
$$

[Here $\Delta \equiv \Delta(\boldsymbol{k} ; \boldsymbol{p}, \boldsymbol{q})$ means the set of all $\boldsymbol{p}$ 's and $\boldsymbol{q}$ 's such that $\boldsymbol{k}+\boldsymbol{p}+\boldsymbol{q}=0$.] A problem is that solutions of the DIA are very difficult to obtain.

An alternative procedure is to directly consider the expression for the turbulent damping rate $\eta_{\boldsymbol{k}}$ that follows from a Markovian statistical closure approximation. For example, in the eddy-damped quasi-normal Markovian (EDQNM) approximation one has

$$
\eta_{k}=-\sum_{\Delta} M_{k p q} M_{p q k}^{*} \theta_{k p q}^{*} C_{q}
$$

where $\theta_{\boldsymbol{k} \boldsymbol{p} \boldsymbol{q}} \doteq\left(\eta_{\boldsymbol{k}}+\eta_{\boldsymbol{p}}+\eta_{\boldsymbol{q}}\right)^{-1}$ is the steady-state triad interaction time. If diffusion is appropriate, then $\mu=$ $\lim _{k \rightarrow 0} \eta_{\boldsymbol{k}} / k^{2}$; if that limit is finite, one concludes that $C_{L}$ is integrable. To illustrate, I consider the expression for the turbulent damping rate (not the density diffusion coefficient) in the undriven Hasegawa-Mima equation: ${ }^{42}$

$$
\begin{aligned}
\eta_{\boldsymbol{k}}=-\sum_{\Delta} & \frac{|\widehat{\boldsymbol{z}} \cdot \boldsymbol{p} \times \boldsymbol{q}|^{2}\left(p^{-2}-q^{-2}\right)\left(q^{-2}-k^{-2}\right)}{\left(\left[k^{-2}\right]+1\right)\left(\left[p^{-2}\right]+1\right)} \\
& \times\left(\eta_{\boldsymbol{k}}^{*}+\eta_{\boldsymbol{p}}^{*}+\eta_{\boldsymbol{q}}^{*}\right)^{-1} C_{\boldsymbol{q}} .
\end{aligned}
$$

The bracketed terms in the denominator stem from adiabatic electron response; when they are omitted, the model reduces to the 2D guiding-center model. ${ }^{43}$ For simplicity, I shall assume homogeneity, even though that is not strictly valid for nonzero diamagnetic velocity $V_{\star}$; alternatively, one may simply assume thermal equilibrium, for which $V_{\star}$ rigorously vanishes. Then

$$
\begin{gathered}
\eta_{\boldsymbol{k}}=-\frac{2}{(2 \pi)^{2}} \int_{\Delta} d p d q p^{2} q^{2}|\sin \alpha| \frac{\left(p^{-2}-q^{-2}\right)\left(q^{-2}-k^{-2}\right)}{\left(\left[k^{-2}\right]+1\right)\left(\left[p^{-2}\right]+1\right)} \\
\times\left(\eta_{k}^{*}+\eta_{p}^{*}+\eta_{q}^{*}\right)^{-1} C_{q},
\end{gathered}
$$

where $\alpha=\angle(\boldsymbol{p}, \boldsymbol{q})$ and the domain $\Delta(k ; p, q)$ is depicted, for example, in Fig. 5.5.1 of Ref. 21. Note that $C_{q}=q^{2} V_{q}$, where $V_{q}$ is the velocity spectrum. I shall consider two spectral forms for $C_{q}$ : a "ring" model in which the spectrum is concentrated in an energy-containing range such that $p, q \gg k$ as $k \rightarrow 0$; and a thermal-equilibrium model, in which $V_{q}=\left(a+b q^{2}\right)^{-1}$, where $a$ and $b$ are constants.

In the ring model, one has $\sin \alpha=\mathcal{O}(k), p \sim q$ (so one can write $p=q+\epsilon k$, where $-1 \leq \epsilon \leq 1)$. It is then straightforward to verify that $\eta_{k}=\mathcal{O}\left(-k^{4}\right)$. [One power of $k$ comes from the $p$ integration (of extent $k$ ), one from $\sin \alpha$, and two from $p^{-2}-q^{-2}$ [the $\mathcal{O}(k)$ term vanishes by symmetry]. A definite calculation that retains numerical coefficients can be done by postulating the shell spectrum $C_{q}=(2 \pi q)^{-1} \delta(q-\bar{q}) \bar{C}$.] The power of $k^{4}$ is consistent with the expectation of an eddy viscosity acting on vorticity $\omega$. [One must remember that the dependent variable in the Hasegawa-Mima equation is the potential $\varphi$, for which vorticity diffusion is represented as $k^{2} \times k^{2}$, where the first $k^{2}$ arises from the diffusion law and the second $k^{2}$ arises from $\omega=\nabla^{2} \varphi$. The divisors in formulas such as Eq. (20b) must be modified to $k^{4}$ for this case.] Thus one concludes that the vorticity viscosity coefficient $\mu$ is finite and $C_{L}$ is integrable. That $\mu$ is negative is consistent with known results for eddy viscosity in systems with two conserved quantities, ${ }^{44}$ which possess inverse energy cascades.

In thermal equilibrium, one can follow the outlines of the guiding-center calculation performed in Ref. 43. Upon noting denominator terms of the form $1+p^{2}$ or $a+b q^{2}$, one can deduce that a large-wave-number cutoff exists; further inspection shows that the dominant contributions come from wave numbers of $\mathcal{O}(k)$. If one neglects small terms and assumes that $V_{q}=$ const., one can symmetrize (24) to find 


$$
\eta_{k}=\frac{1}{(2 \pi)^{2}} \int_{\Delta} d p d q|\sin \alpha|\left(p^{2}-q^{2}\right)^{2} \frac{V}{\eta_{k}+\eta_{p}+\eta_{q}},
$$

a manifestly positive form that can be shown to be $\propto k^{4}$. Analysis shows that $\eta_{k}$ is convergent, so again one concludes that $C_{L}$ is integrable.

If the adiabatic electron response is ignored, one recovers the guiding-center plasma model and the results of Ref. 43, namely that $\eta_{k}=k^{2} \mu_{k}$, where $\mu_{k}$ is positive and involves the square root of the logarithmic divergence $\ln \left(k^{-1}\right)$. That divergence is the remnant of the $\tau^{-d / 2}$ tail for $d=2$, which for the guiding-center model only contaminates the Lagrangian correlation function as well as the Eulerian one. Evidently, whereas slow decay of $C_{E}$ is quite general, the behavior of $C_{L}$ depends on subtle details of wave-number distributions and physical assumptions. One must not infer properties of transport coefficients from Eulerian functions.

\section{SUMMARY}

I have (i) discussed the use of stochastic models to provide additional perspectives on the statistical behavior of turbulent systems, and mentioned a simple model that produces long-time Eulerian tails although it is not SOC-like and has quasilinear transport coefficients; (ii) reviewed the spectral balance equation and described how a formula for the frequency spectrum of uncorrelated overlapping avalanches is a special case of that equation; (iii) argued that there need be no contradiction between observations of long-time tails on Eulerian correlation functions and the existence of local transport coefficients.

This work does not specifically address the possible role of avalanches in creating the observable fluctuations and transport, or the possibility that actual transport is in fact nondiffusive. ${ }^{45}$ In general turbulence theory, avalanches are not singled out for special treatment; they are simply random fluctuations with a particular (threshold-related) driving mechanism. Of course, specific properties of such fluctuations (e.g., intermittent PDF's ${ }^{7}$ ) are of considerable interest, particularly in submarginal scenarios ${ }^{46}$ (although transport need not depend strongly on the details of higherorder statistics ${ }^{47}$ ). In any case, further work must be done to elucidate the actual nonlinear dynamics underlying the experimental observations, to determine whether specific results or theories about the asymptotic SOC limit are useful, and to obtain a unified formalism that quantitatively describes all regimes of turbulence and transport.

\section{ACKNOWLEDGMENTS}

The work summarized in Sec. II was performed in collaboration with M. Ottaviani. I have enjoyed helpful discussions with B. Carreras, T.-S. Hahm, Z. Lin, and W. Nevins. This research was supported by the U.S. Department of Energy Contract No. DE-AC02-76-CHO-3073.
${ }^{1}$ P. Bak, C. Tang, and K. Wiesenfeld, Phys. Rev. Lett. 59, 381 (1987), Phys. Rev. A 38, 364 (1988).

2 P. H. Diamond and T.-S. Hahm, Phys. Plasmas 2, 3640 (1995).

${ }^{3}$ D. E. Newman, B. A. Carreras, P. H. Diamond, and T.-S. Hahm, Phys. Plasmas 3, 1858 (1996)

${ }^{4}$ B. A. Carreras, D. Newman, V. E. Lynch, and P. H. Diamond, Plasma Phys. Rep. 22, 740 (1996).

${ }^{5}$ D. Newman, Bull. Am. Phys. Soc. 44, 182 (1999).

${ }^{6}$ B. A. Carreras, D. Newman, V. E. Lynch, and P. H. Diamond, Phys. Plasmas 3, 2903 (1996).

7 B. A. Carreras, B. van Milligen, C. Hidalgo et al., Phys. Rev. Lett. 83, 3653 (1999).

8 P. A. Politzer, Observation of avalanche-like phenomena in a magnetically confined plasmas, Phys. Rev. Lett. (1999) (in press).

${ }^{9}$ W. M. Nevins, Bull. Am. Phys. Soc. 44, ??? (1999).

10 B. A. Carreras, B. van Milligen, M. A. Pedrosa et al., Phys. Rev. Lett. 80, 4438 (1998).

${ }^{11}$ B. A. Carreras, B. P. van Milligen, M. A. Pedrosa et al., Phys. Plasmas 5, 3632 (1998).

12 B. A. Carreras, Bull. Am. Phys. Soc. 43, 1920 (1998).

13 B. A. Carreras, D. E. Newman, B. P. van Milligen, and C. Hidalgo, Phys. Plasmas 6, 485 (1999).

14 A. Vespignani and S. Zapperi, Phys. Rev. E 57, 6345 (1988).

${ }^{15}$ R. Cafiero, V. Loreto, L. Pietronero, A. Vespignani, and S. Zapperi, Europhys. Lett. 29, 111 (1995).

16 R. V. Jensen, J. Stat. Phys. 25, 183 (1981).

17 J. A. Krommes and M. Ottaviani, Phys. Plasmas 6, 3731 (1999).

18 For some discussion of stochastic models that support submarginal profiles, see J. A. Krommes, Phys. Plasmas 4, 1342 (1997).

19 H. J. Jensen, Self-Organized Criticality (Cambridge University Press, Cambridge, UK, 1998).

20 P. C. Martin, E. D. Siggia, and H. A. Rose, Phys. Rev. A 8, 423 (1973).

21 J. A. Krommes, in Handbook of Plasma Physics, edited by A. A. Galeev and R. N. Sudan (North-Holland, Amsterdam, 1984), Vol. 2, Chap. 5.5, p. 183.

22 J. A. Krommes, Renormalized dissipation in the nonconservatively forced Burgers equation, submitted to Phys. Plasmas.

23 J. A. Krommes, The influence of random zonal flows on saturation levels in simple stochastic models, submitted to Phys. Plasmas.

${ }^{24}$ C. E. Leith, J. Atm. Sci. 28, 145 (1971).

25 R. H. Kraichnan, J. Fluid Mech. 41, 189 (1970).

26 J. A. Krommes, Phys. Rev. E 53, 4865 (1996).

27 G. E. Uhlenbeck and L. S. Ornstein, Phys. Rev. 36, 823 (1930), Reprinted in Selected Papers on Noise and Stochastic Processes, edited by N. Wax (Dover, New York, 1954), p. 93.

28 J. Bowman, J. A. Krommes, and M. Ottaviani, Phys. Fluids B 5, 3558 (1993)

29 J. A. Krommes and R. A. Smith, Ann. Phys. (NY) 177, 246 (1987).

${ }^{30}$ R. Kubo, in Lectures in Theoretical Physics, edited by W. E. Brittin and L. G. Dunham (Interscience, New York, 1959), Vol. I, p. 181.

${ }^{31}$ R. H. Kraichnan, J. Math. Phys. 2, 124 (1961), erratum: J. Math. Phys. 3, 205 (1962).

32 R. Kubo, in Fluctuation, Relaxation, and Resonance in Magnetic Systems, edited by D. ter Harr (Oliver and Boyd, Edinburgh, 1962), p. 23.

33 G. I. Taylor, Proc. London Math. Soc., Ser. 2 20, 196 (1921), reprinted in Turbulence: Classic Papers on Statistical Theory, edited by S. K. Friedlander and L. Topper (Interscience, New York, 1961), p. 1.

${ }^{34}$ J. L. Lumley, in Mechanique de la Turbulence (Editions du Centre National de la Recherche Scientifique, Paris, 1962), p. 17.

35 J. R. Herring, Phys. Fluids 8, 2219 (1965).

36 J. A. Krommes and C. Oberman, J. Plasma Phys. 16, 193 (1976).

37 R. H. Kraichnan, Phys. Rev. 113, 1181 (1959).

38 J. A. Krommes, Phys. Fluids B 5, 650 (1993).

39 B. J. Alder and T. E. Wainwright, Phys. Rev. A 1, 18 (1970).

40 J. A. Krommes and C. Oberman, J. Plasma Phys. 16, 229 (1976).

${ }^{41}$ L. P. Kadanoff and P. C. Martin, Ann. Phys. 24, 419 (1963). 
42 J. C. Bowman and J. A. Krommes, Phys. Plasmas 4, 3895 (1997).

43 J. A. Krommes and P. Similon, Phys. Fluids 23, 1553 (1980).

${ }^{44}$ R. H. Kraichnan, J. Atm. Sci. 33, 1521 (1976).

45 B. A. Carreras, Bull. Am. Phys. Soc. 44, ??? (1999).
46 J. A. Krommes, Plasma Phys. Control. Fusion 41, A641 (1999).

47 G. Hu, J. A. Krommes, and J. C. Bowman, Phys. Plasmas 4, 2116 (1997). 